\title{
Ejercicio efectivo de los derechos humanos de los adolescentes en la escuela: la versión docente
}

\author{
Effective exercise of the human rights of adolescents \\ at school: the teaching version
}

\author{
José María Nava-Preciado ${ }^{1}$ \\ Arturo Villa-Flores ${ }^{2}$
}

\begin{abstract}
Resumen: Este trabajo tiene como objetivo explorar algunas posiciones de los docentes sobre los derechos humanos de los adolescentes, tomando como marco la Ley General de los Derechos de Niñas, Niños y Adolescentes en México, para valorar si dichas posturas implican un conocimiento efectivo o deficitario de dicha ley. Es un estudio de carácter mixto que tiene como muestra a 84 docentes de educación secundaria de una zona escolar de Guadalajara, México. Los datos se recuperan a través de un cuestionario que incluye aspectos objetivos y también subjetivos. El análisis permite la descripción de datos cuantitativos así como la comprensión del sentido mentado por los actores. Los resultados preliminares arrojan que los docentes tienen un conocimiento marginal de la Ley que moldea, en el fondo y en la forma, sus concepciones sobre los derechos humanos de los adolescentes, entre otras razones porque no reciben capacitación en esta materia.
\end{abstract}

Palabras clave: adolescente, personal docente, derechos humanos, juicio, centro de enseñanza secundaria.

\footnotetext{
1 Doctor en Educación. Profesor titular de la Universidad de Guadalajara. Miembro del Sistema Nacional de Investigadores nivel I. México. E-mail: jnava_preciado@yahoo.com.mx.

2 Doctor en Ciencias Sociales. Profesor Asociado de la Universidad de Guadalajara, México.

E-mail: arturovillaflores@yahoo.com.
}

Diálagos Pedagágicas. ISSN en línea: 2524-9274

Año XVI, No 32, octubre 2018-marzo 2019. Pág. 43-61

DOI: http://dx.doi.org/10.22529/dp.2018.16(32)03 / Recibido: 12-06-2018 / Aprobado: 06-08-2018

(i) $\odot$ Artículo publicado bajo Licencia Creative Commons Atribución-NoComercial-SinDerivar. (C) Universidad Católica de Córdoba. 


\begin{abstract}
This work aims to explore some positions of teachers on the human rights of adolescents, taking as a framework the General Law on the rights of girls, children and adolescents in Mexico, to assess whether these positions imply an effective or deficient knowledge of that law. It is a mixed study that shows 84 secondary education teachers in a school area in Guadalajara, Mexico. The data is recovered through a questionnaire that includes objective and also subjective aspects. The analysis allows the description of quantitative data as well as the understanding of the sense of the actors. Preliminary results show that teachers have a marginal knowledge of the law, which shapes, in the background and in the form, their conceptions about the human rights of adolescents, among other reasons because they do not receive proper training in this area.
\end{abstract}

Key words: adolescent, teaching personnel, human rights, judgement, secondary school.

\title{
Introducción
}

Los adolescentes están expuestos a una serie de violencias propias de una sociedad cada vez más competitiva y tecnificada pero, al mismo tiempo, más desocializada (Touraine, 1997) e insensible al sufrimiento humano (Bauman \& Donskis, 2015). Por ejemplo, en nuestro contexto algunos adolescentes viven situaciones que van desde la desaparición ${ }^{3}$ hasta alguna forma de violencia ${ }^{4}$ desde la deserción escolar ${ }^{5}$ hasta los embarazos a edades tempranas. ${ }^{6}$ Estos problemas se presentan en diferentes contextos sociales y culturales, y evidencian en todos los casos las condiciones de vulnerabilidad de este sector de la población. Estas historias de maltrato, tan repetidas y escuchadas, llevaron al legislativo mexicano a promulgar la Ley General de los Derechos de Niñas, Niños y Adolescentes [LGDNNA] (México, Comisión Nacional de los Derechos Humanos, 2015).7

\footnotetext{
${ }^{3}$ Por ejemplo, según nota de El Informador (Adolescentes, las que más desaparecen en la entidad, 2016), en Jalisco las mujeres adolescentes constituyen el grupo de población que más sufre la desaparición. De cada diez casos que se presentan, seis corresponden a adolescentes de entre 15 y 18 años, según datos de la Fiscalía General del Estado.

${ }^{4}$ La Encuesta Nacional de Salud y Nutrición 2012 (2012) reporta que el 3.9\% de un total de 21.519 adolescentes entrevistados sufrió robo, agresión o algún tipo de violencia. Mientras tanto, la Encuesta de Cohesión Social para la Prevención de la Violencia y la Delincuencia 2014 (2015) arroja que el $4.5 \%$ de los jóvenes que estudian y trabajan estima que sus maestros y/o jefes los han maltratado.

${ }^{5}$ Según la Encuesta Nacional de la Juventud 2010 (2011), del total de adolescentes en el país, el 6\% ni estudiaba ni trabajaba. Estos son datos del 2010, pero constituyen un indicador de lo que pudiera estar pasando actualmente con este grupo de población. La encuesta muestra que a partir de los 15 años la asistencia a la escuela empieza a disminuir de manera sensible.

${ }^{6}$ De acuerdo a la UNICEF México, nuestro país ocupa el primer lugar en el índice de embarazos adolescentes dentro de los países de la OCDE, con 64 casos por cada mil (Instituto Nacional de Salud Pública y UNICEF México, 2016).

7 La ley fue promulgada el 4 de diciembre de 2014; con ella se creó así una nueva institucionalidad para dar cumplimento a los derechos de la infancia y adolescencia sin precedentes en el país, según González (2016).
} 
Sin duda, dos razones fueron determinantes para su promulgación: 1) la emergencia de nuevas formas de violencia en contra de las y los adolescentes, ${ }^{8}$ que hace ineludible legislar para inhibirlas; 2) la urgencia por luchar para que los dividendos de los derechos humanos (DD.HH.) de este grupo de población se instalen definitiva e irrestrictamente en nuestra sociedad. A nuestro análisis subyace la idea de que esta ley tiene un carácter positivo y uno negativo. Su índole positiva radica en el establecimiento de los derechos a un libre desarrollo que, de acuerdo a su edad, los agentes mencionados tienen y, por tanto, deben ser protegidos. Su condición negativa se focaliza en el rechazo a cualquier acción, intervención o medida de personas o instituciones, que vulneren estos derechos.

Los adolescentes no son iguales en capacidades físicas e intelectuales a un adulto; ${ }^{9}$ tampoco lo son en cuanto a capacidades morales, bajo el supuesto de que alcanzarán, en un estadio ulterior, su maduración cognitiva y ética (Durkheim, 2002). Este reconocimiento implica que la sociedad debe ser garante de las condiciones mínimas y suficientes que protejan los derechos de este grupo social (México, Comisión Nacional de los Derechos Humanos, 2015). ¿Cómo debemos valorar la LGDNNA? Como una meta-ley, como un andamiaje jurídico valioso de gran envergadura que moldea los DD.HH. de los adolescentes. Sin embargo, su promulgación no puede quedar en una dimensión meramente formal y discursiva. Su contenido debe atender de manera efectiva e irrestricta en términos materiales los problemas que vive este segmento de población. ¿Qué significa esto? Que los debates de una ley con este peso moral y jurídico no pueden quedarse solo en el plano retórico; al contrario, las deliberaciones deben dirigirse a las formas concretas de su aplicación, porque los derechos se cumplen cuando los destinarios los disfrutan (Amar, Madariaga \& Macías, 2007).

Por lo tanto, los DD.HH. de los adolescentes deben constituirse en una praxis social de concientización de los agentes que interactúan cotidianamente con ellos. Así, nos preguntamos: ¿quiénes son los destinatarios de esta ley? ¿Los adolescentes? ¿La sociedad? ¿Los padres de familia? Al respecto, la mencionada ley afirma en su artículo 11:

Es deber de la familia, la comunidad a la que pertenecen, del Estado $y$, en general, de todos los integrantes de la sociedad, el respeto y el auxilio para la protección de derechos de niñas, niños y adolescentes, así como garantizarles un nivel adecuado de vida.

Si observamos detenidamente el contenido de ese artículo, encontramos que la escuela, al ser un espacio comunitario donde se interactúa naturalmente con los adolescentes, también está obligada a garantizar la observancia de los DD.HH.,

\footnotetext{
${ }^{8}$ Nuestro estudio se focaliza en centros de enseñanza secundaria, por esta razón solo hacemos referencia a los adolescentes en el contexto de la LGDNNA.

9 Esto no quiere decir que los adolescentes sean incapaces, sino que tienen solo una limitación en cuanto a su capacidad de obrar, la cual se entiende como necesaria para proteger sus derechos, tal y como lo sostiene Matus (2013).
} 
pues es una obligación de todas las personas y no solo del Estado (Organización de las Naciones Unidas, México, 2008). Asimismo, en su artículo 43, la LGDNNA establece que los adolescentes tienen derecho a vivir en un ambiente sano y sustentable que favorezca su desarrollo integral; esto significa que la escuela debe ser un espacio garante de ese derecho, porque en el contexto escolar se imponen una serie de normas, valores y pautas de funcionamiento que, de forma íntima y subjetiva (Fuentes, González, Padrón \& Tapia, 2015), moldean sus derechos y, en algún sentido, materializan lo que conocemos como la institucionalización de los DD.HH. (Ansolabehere \& Navarrete, 2015).

De ahí la formulación de las siguientes preguntas: ¿los docentes tienen conocimiento de la ley? ¿Promueven los DD.HH. de sus alumnos? ¿Son sensibles al tema de los DD.HH. de los adolescentes en la escuela? Dadas estas interrogantes, el objetivo de este escrito es explorar algunas posiciones de los profesores sobre los DD.HH. de los adolescentes, tomando como marco la LGDNNA, de tal manera que nos permita valorar si dichas posturas implican un conocimiento efectivo o deficitario de la ley en mención. El tratamiento del ejercicio efectivo de los DD.HH. busca sopesar qué tantas dificultades tiene la escuela por un lado, para reconocerlos y, por el otro, para aplicarlos. En este sentido más que teorizar sobre su importancia en el ámbito escolar, que es una tarea ineludible, nuestra preocupación está centrada en analizar su aplicación en espacios concretos. Toda discusión sobre la pertinencia o no de una ley se puede volver vacía si no damos cuenta de su efectiva vigencia.

Es importante señalar que el presente estudio es un aporte modesto que recupera únicamente las valoraciones y la experiencia de uno de los destinatarios de la ley: los docentes, sin dejar de reconocer que el otro grupo son los propios adolescentes.

Por ello, el supuesto del que parte este trabajo es que la comunidad de profesores es una comunidad que comparte y crea experiencias escolares, construyendo bajo esta premisa un performance narrativo de cómo se deben cultivar los $\mathrm{DD} . \mathrm{HH}$. de sus alumnas y alumnos en la escuela, realizando esta tarea a partir de sus propias intuiciones y no tanto de un conocimiento pleno de la LGDNNA.

\section{Aproximaciones metodológicas}

Lógica de estudio. La manera en cómo se problematiza un objeto de estudio, se recupera la información y se hace la faena de interpretación define en su conjunto los supuestos epistemológicos de una investigación, sin ánimos reduccionistas (Páramo, 2013). Esto implica tomar decisiones metodológicas vinculadas a asegurar una mejor comprensión del problema y elaborar un diseño de investigación (Yuni \& Urbano, 2014). Partiendo de esta perspectiva, y asociando el objetivo de estudio con los métodos y técnicas específicas de trabajo (Ragin, 2007), nuestra investigación se hace desde una perspectiva que involucra datos cuantitativos y cualitativos; es decir un acercamiento mixto. Este enfoque nos permite cierta flexibilidad tanto en la propuesta escrita como en el diseño de la investigación. Conviene señalar que por sus alcances nuestro trabajo es de carácter exploratorio, 
porque en este tipo de estudios la información es preliminar, se identifican variables relevantes y se formulan hipótesis de las relaciones entre ellas (Páramo, 2013; Yuni \& Urbano, 2014).

Sujetos. Flick (2007) argumenta que si nos posicionamos en una perspectiva preferentemente cualitativa el muestreo se dirige a personas o grupos que brindan información relevante para los propósitos de la investigación. Así, con base en una lógica de conveniencia y accesibilidad (Hernández, Fernández \& Baptista, 2014), en este muestreo intencionado elegimos como unidades de observación a docentes de secundarias públicas del municipio de Guadalajara. Se hizo así, entre otras razones, por ser destinatarios indirectos de la LGDNNA y tener el deber de respetar, promover y observar los DD.HH. de sus alumnos.

Elección de las escuelas. Una vez definidos los sujetos, establecimos contacto con el inspector de una zona escolar a quien se le presentó el proyecto de investigación. Después de un diálogo sobre la problemática adolescente, se nos solicitó presentarlo a los directores de sus escuelas en una reunión de trabajo. Una vez que los directivos se sensibilizaron sobre la pertinencia del estudio, el inspector nos propuso que el trabajo de campo se efectuara con docentes provenientes de seis centros escolares que forman parte de la zona escolar. Cabe aclarar que estas escuelas están ubicadas en la periferia de la ciudad. Como se puede observar, el muestreo es de carácter accidental. En este tipo de muestreo, según argumentan Yuni y Urbano (2014) "se seleccionan los casos por su capacidad de generar información relevante" (p. 14).

Técnica para recolectar los datos. En la investigación en ciencias sociales las técnicas se adecuan a los objetivos de estudio y a los requerimientos de cada situación (Hernández et al., 2014). Con base en esta premisa optamos por elaborar un instrumento apropiado que nos permitiera obtener los datos de una manera práctica, encontrando en el cuestionario ${ }^{10}$ una técnica de registro idónea para acercarnos a los docentes frente a grupo. Se puede decir que, recuperando una propuesta de Cea (2001), el cuestionario incluye aspectos objetivos (hechos) y también subjetivos (juicios valorativos); asimismo, algunas preguntas se aplican con base en la técnica de listas libres, con el propósito de revisar la importancia que le dan los entrevistados a cierto dominio, así como su nivel de significancia, que es una función entre la frecuencia de mención y el rango o importancia atribuida por el sujeto (Romney, Weller \& Batchelder, 1986). Esta estrategia de recolección de información permite obtener información cuantitativa por medio de un cuestionario, así como información cualitativa obtenida a través de preguntas abiertas y una técnica de listas libres que permite vincular

\footnotetext{
10 Nuestro cuestionario tomó como referente La Encuesta Nacional de Niños, Adolescentes y Jóvenes (ver Fuentes et al., 2015) que investigadores de la UNAM utilizaron en el estudio "Los mexicanos vistos por sí mismos. Los grandes temas nacionales". Con la finalidad de asegurar la validez y la confiabilidad del instrumento se sometió a la consideración de dos expertos. Ya con la versión final, aplicamos un pilotaje a 15 profesores de una escuela secundaria, ubicada en un contexto similar a las escuelas seleccionadas.
} 
datos cualitativos y cuantitativos. La interpretación de la información por supuesto se realiza desde un enfoque preponderantemente cualitativo, es decir se trata de captar las conexiones de sentido que atribuyen los sujetos a la acción social y las posibles relaciones de causalidad adecuada entre hechos culturales y acciones sociales (Weber, 2009).

Protocolo. La mejor estrategia para recuperar la información con prontitud y efectividad con el personal docente fue aprovechar una sesión de trabajo convocada por el inspector de la zona escolar. Con este grupo docente convenimos la importancia de su participación después de aclarar los propósitos del estudio. Solo se recuperaron 84 cuestionarios completos de los 119 administrados. El 53.5\% de los encuestados tiene de 16 a 25 años de servicio; por su parte, el 52\% de ellos eran hombres y el $48 \%$ mujeres.

Análisis de la información. Los datos se analizaron escuela por escuela tomando en cuenta el género y los años de servicio de los docentes. Como lo sugieren Yuni y Urbano (2014), este subtipo de triangulación nos permitió un análisis de cada respuesta. Así, la triangulación, reevaluación y discusión, informante por informante y plantel por plantel, nos posibilitó construir una serie de categorías con los contenidos de la sección cualitativa del instrumento. Por su parte, el contenido que sirve para distribuir frecuencias se muestra y se agrupa en tablas y porcentajes con relación a cada categoría de análisis. Esto es, se busca hacer un cruce entre datos cuantitativos y cualitativos, recuperando los planteamientos de Hernández, Fernández y Baptista (2014), enfatizando la interpretación de acuerdo a nuestra lógica de estudio.

\section{Resultados}

Para el estudio, partimos de suponer el conocimiento de los docentes en torno a la promulgación de la LGDNNA, de ahí la pertinencia de preguntarles, de acuerdo a su experiencia, el estado que guarda el ejercicio efectivo de los DD.HH. de los adolescentes. Para el $55.95 \%$ de los investigados los DD.HH. han mejorado, mientras que para el $23.81 \%$ siguen igual y el $20.24 \%$ considera que han empeorado. Algunas razones que encontramos en el primer posicionamiento son: "Creo que han mejorado, porque hoy día se pueden ver más libertades en ellos, más protección y mejores cuidados de los padres o tutores, así como de los docentes" (Prof. 32); "Porque hay más conciencia, tanto en la sociedad civil como en la escuela" (Profa. 64). Por su parte, entre las narrativas que observamos en el segundo grupo encontramos este tipo de construcciones: "Sólo se dice de los cambios, pero en general todo ha seguido de la misma manera" (Prof. 69); "Es lo que demuestran las estadísticas y no está vinculado a la existencia de una ley sino a las condiciones socioeconómicas y culturales" (Profa. 51). En cuanto al tercer grupo, se esgrimen puntos de vista como el siguiente: "Han empeorado, porque la juventud actual ni estudia ni trabaja, carecen de valores, no tienen una visión a futuro, los problemas de vandalismo y drogadicción los ha afectado bastante" (Prof. 46); "Han empeorado, porque sólo protegen al adolescente y al maestro no, nunca investigan más a fondo la raíz del problema" (Profa. 12). 
Para abordar qué tanto la escuela respeta los DD.HH., preguntamos a los docentes su apreciación al respecto, considerando una escala de cuatro categorías ( $m u c h o=1 ;$ poco $=2 ;$ algo $=3 ;$ nada $=4$ ). Sus repuestas tienen un comportamiento similar a los resultados de la pregunta anterior. Por ejemplo, el $58.33 \%$ piensa que en la escuela se respetan mucho los DD.HH.; por su parte el $14.29 \%$ considera que poco y el $22.61 \%$ cree que algo; solo el $3.57 \%$ está convencido que nada se respetan. Poniendo en relación estos datos con las narrativas que sostienen las posturas correspondientes, encontramos lo que sigue: Mucho: "Son el motor de la escuela" (Profa. 18); "Se vigilia que en su mayoría los adolescentes tengan prioridad ante todo" (Profa. 37); "Estamos al cuidado de los alumnos" (Prof. 04); "La mayoría de los profesores les damos buen trato" (Prof. 11); "Un docente que organiza y evalúa su quehacer educativo y lleva a cabo una intervención didáctica pertinente fomenta en sus alumnos derechos y obligaciones" (Profa. 09). Poco: "Porque no se conocen, porque hay un temor" (Prof. 55); "Algunas veces son ignorados a conveniencia" (Prof. 76). Algo: "Los maestros desconocemos sus derechos y no sabemos cómo manejarlos" (Prof. 07); "Porque no se ha logrado abatir la desigualdad de género" (Prof. 28). Nada: "Hay muchas faltas a la moral entre adolescentes y no se hace nada" (Profa. 44).

Sin embargo, paradójicamente, nos percatamos de que los docentes no toleran algunas conductas o gustos personales de los adolescentes (ver Tabla 1). Esto implica necesariamente una tensión, porque dicen, por un lado, que en la escuela se respetan mucho los DD.HH. y, por el otro, un alto porcentaje de ellos rechaza algunos hábitos que cultivan los adolescentes.

Los hábitos mencionados son tema de tensión cotidiana entre docentes y estudiantes $y$, como se observa en la Tabla 1, los profesores no están dispuestos a tolerarlos, aunque en una escala de cuatro categorías (mucho $=1 ; \operatorname{poco}=2 ;$ algo $=3$;

Tabla 1. El grado de aceptación por el profesorado de algunas conductas adolescentes

\begin{tabular}{|l|c|c|c|c|c|c|c|c|}
\cline { 2 - 10 } \multicolumn{1}{c|}{} & \multicolumn{8}{c|}{ Valor de la etiqueta } \\
\cline { 2 - 10 } \multicolumn{1}{c|}{} & \multicolumn{2}{c|}{ Mucho } & \multicolumn{2}{c|}{ Algo } & \multicolumn{2}{c|}{ Poco } & \multicolumn{2}{c|}{ Nada } \\
\hline Ítem & $\mathbf{N}^{\circ}$ & $\mathbf{\%}$ & $\mathbf{N}^{\circ}$ & $\mathbf{\%}$ & $\mathbf{N}^{\circ}$ & $\%$ & $\mathbf{N}^{\circ}$ & $\%$ \\
\hline Tatuajes en el cuerpo & 5 & 5.95 & 7 & 8.33 & 13 & 15.48 & 56 & 66.67 \\
\hline Maquillaje en mujeres & 4 & 4.76 & 12 & 14.29 & 19 & 22.62 & 58 & 57.14 \\
\hline $\begin{array}{l}\text { Cortes modernos de } \\
\text { pelo en los hombres }\end{array}$ & 5 & 5.95 & 19 & 22.62 & 18 & 21.43 & 40 & 47.62 \\
\hline Faldas cortas & 8 & 9.52 & 13 & 15.48 & 14 & 16.67 & 46 & 54.76 \\
\hline $\begin{array}{l}\text { Uso de celulares } \\
\text { en la escuela }\end{array}$ & 11 & 13.10 & 23 & 27.38 & 20 & 23.81 & 28 & 33.33 \\
\hline
\end{tabular}

Fuente: elaboración propia. 
nada $=4$ : no sé $=5$ ), el $72.62 \%$ considera que la opinión de los estudiantes es importante en los asuntos escolares donde éstos se ven implicados. Para sostener sus posiciones esgrimen argumentos como los siguientes: "Al involucrarlos se sienten parte de los problemas y dan mejores resultados" (Prof. 36); "Porque así tiene más significado el sentido de la escuela" (Profa. 72); "Porque es su derecho y su opinión refleja cómo viven su realidad" (Prof. 81); "Porque de esa manera conocemos sus inquietudes, necesidades y gustos $y$, al conocerlos, podemos motivarlos más" (Profa. 19); "Son ellos los que viven esta etapa y nos pueden guiar en cómo pueden ser tratados para una mejor relación entre profesores y alumnos y así ellos logren un mejor aprendizaje" (Profa. 24). Los valores de nada y no sé se ubicaron en cero por ciento, mientras que las categorías de algo y poco en su conjunto alcanzaron un $27.38 \%$.

Para conocer el nivel de relevancia y el orden que les atribuyen a las principales dificultades por las que los adolescentes no participan en las actividades de la escuela, aplicamos la técnica de listas libres (ver Tabla 2). Encontramos que la mención Los padres de familia no escuchan a los adolescentes presenta un nivel de significancia de .0794; mientras que el índice de prominencia es de 1.91. La siguiente lista con mayor relevancia es que Los adolescentes no tienen capacidad para participar; aquí, el nivel de mención arroja una significancia de 0.717 , la cual es, sin duda, muy alta. Pero en un índice de significancia menor se encuentra la mención: Los profesores no escuchan a los adolescentes. Así, los docentes se atribuyen poca responsabilidad en este tópico.

De la no participación en las actividades, nos dirigimos a ciertas conductas de los jóvenes que son resultado de un entorno vulnerable y que requieren un tratamiento cuidadoso. Algunas de ellas, de acuerdo a una mayoría de docentes, no se justifican en la escuela, ameritando así la expulsión de los alumnos (ver Tabla 3).

Tabla 2. Principales dificultades por las cuales los adolescentes no participan en las actividades de la escuela

\begin{tabular}{|l|c|c|}
\hline Mención identificada & $\begin{array}{c}\text { Rango de } \\
\text { prominencia }\end{array}$ & $\begin{array}{c}\text { Correlación } \\
\text { sobresaliente }\end{array}$ \\
\hline $\begin{array}{l}\text { Los padres de familia no escuchan } \\
\text { a los adolescentes }\end{array}$ & 1.91 & 0.794 \\
\hline $\begin{array}{l}\text { Los adolescentes no tienen } \\
\text { capacidad para participar }\end{array}$ & 2.34 & 0.717 \\
\hline $\begin{array}{l}\text { Los adolescentes quieren tomar } \\
\text { decisiones que no les corresponden }\end{array}$ & 2.87 & 0.613 \\
\hline $\begin{array}{l}\text { Los adolescentes no están } \\
\text { interesados en participar }\end{array}$ & 3.54 & 0.470 \\
\hline $\begin{array}{l}\text { Los profesores no escuchan a } \\
\text { los adolescentes }\end{array}$ & 4.23 & 0.342 \\
\hline
\end{tabular}

Fuente: elaboración propia. 
Estas conductas nos llevan a cuestionar sobre el conocimiento de los jóvenes en torno a sus derechos y obligaciones; así, preguntamos al profesorado si los adolescentes son conscientes de esto. El asunto surge porque existe una idea extendida en el medio escolar que puede resumirse de la siguiente manera: conforme se posiciona el discurso de los DD.HH. de los alumnos, disminuye la autoridad docente. Podemos estimar que el punto de vista de un gran número de estos profesores se asocia con tal idea (ver Tabla 4).

En este orden de ideas, se cuestionó a los docentes qué tanto los estudiantes deben organizarse para exigir sus derechos en la escuela. En una escala de cinco categorías, su posición se concentra, de manera significativa, en los valores mucho y algo (Tabla 5). Entre las razones que apoyan sus posiciones están: "Sí y sólo si tienen en claro qué es lo que exigen, esto es, que sea justo" (Profa. 06); "Sí, para el cumplimiento de sus derechos, pero los adolescentes también deben cumplir por igual sus obligaciones" (Prof. 13); "Los adolescentes tienen el derecho de expresarse, pero también tienen obligaciones de acuerdo con el manual de convivencia" (Prof. 63): "Debemos tomar en cuenta que para exigir derechos deben estar muy conscientes de sus obligaciones" (Profa. 41).

Tabla 3. Conductas que motivan la expulsión de un estudiante, según el profesorado

\begin{tabular}{|l|c|c|c|c|c|c|}
\cline { 2 - 8 } \multicolumn{1}{c|}{} & \multicolumn{6}{c|}{ Valor de la etiqueta } \\
\cline { 2 - 8 } \multicolumn{1}{c|}{} & \multicolumn{2}{c|}{ Acuerdo } & \multicolumn{1}{c|}{ Desacuerdo } & \multicolumn{2}{c|}{ No sé } \\
\hline Ítem & $\mathbf{N}^{\circ}$ & $\mathbf{\%}$ & $\mathbf{N}^{\circ}$ & $\mathbf{\%}$ & $\mathbf{N}^{\circ}$ & $\mathbf{\%}$ \\
\hline Ingiera drogas & 46 & 54.76 & 27 & 32.14 & 9 & 0.71 \\
\hline Ingiera bebidas embriagantes & 34 & 40.48 & 38 & 45.24 & 10 & 11.90 \\
\hline Fume cigarrillos & 17 & 20.24 & 52 & 61.90 & 11 & 13.10 \\
\hline Resulte embarazada & 3 & 3.57 & 78 & 92.86 & 2 & 2.38 \\
\hline $\begin{array}{l}\text { Tenga relaciones sexuales con algún } \\
\text { compañero }\end{array}$ & 20 & 23.81 & 51 & 60.71 & 9 & 10.71 \\
\hline Sea violento con sus compañeros & 50 & 2.38 & 25 & 59.52 & 7 & 29.76 \\
\hline $\begin{array}{l}\text { No respete la disciplina impuesta } \\
\text { por la escuela }\end{array}$ & 40 & 47.62 & 33 & 39.29 & 7 & 8.33 \\
\hline Se enfrente a los profesores & 44 & 52.38 & 26 & 30.95 & 9 & 10.71 \\
\hline Asuma conductas homosexuales & 2 & 2.38 & 75 & 89.29 & 4 & 4.76 \\
\hline Agreda verbalmente a sus compañeros & 14 & 16.67 & 60 & 71.43 & 7 & 8.33 \\
\hline Reprobación sistemática & 15 & 17.86 & 59 & 70.24 & 6 & 7.14 \\
\hline Vean pornografía en clase & 23 & 27.38 & 43 & 51.19 & 14 & 16.67 \\
\hline
\end{tabular}

Fuente: elaboración propia. 
Por su parte, en una escala del 1 al 10 , donde 1 significa que no tienen ninguna preparación para promover los DD.HH. y 10 que tienen suficiente preparación para ello, una gran mayoría de los profesores se consideran lo suficientemente preparados para esta tarea, tal y como se aprecia en la Tabla 6.

Los posiciones de los docentes que se consideran preparados, en la escala de 8 a 10, esgrimen razones como las siguientes: "Constantemente en lo individual nos estamos documentando y lo aplicamos en la práctica" (Prof. 22); "Debemos estar actualizándonos constantemente en el trato adolescente porque ha venido cambiando drásticamente, esto es, debemos conocer a fondo las caracte-

Tabla 4. El conocimiento de los adolescentes sobre sus derechos y obligaciones según los profesores

\begin{tabular}{|c|c|c|c|c|c|c|c|c|c|c|}
\hline \multirow[b]{3}{*}{ Ítem } & \multicolumn{10}{|c|}{ Valor de la etiqueta } \\
\hline & \multicolumn{2}{|c|}{ Acuerdo } & \multicolumn{2}{|c|}{$\begin{array}{l}\text { Acuerdo } \\
\text { en parte }\end{array}$} & \multicolumn{2}{|c|}{ Desac. } & \multicolumn{2}{|c|}{$\begin{array}{l}\text { Desac. } \\
\text { en parte }\end{array}$} & \multicolumn{2}{|c|}{ No sé } \\
\hline & $\mathbf{N}^{\circ}$ & $\%$ & $\mathbf{N}^{\circ}$ & $\%$ & $\mathbf{N}^{\circ}$ & $\%$ & $\mathbf{N}^{\circ}$ & $\%$ & $\mathbf{N}^{\circ}$ & $\%$ \\
\hline $\begin{array}{l}\text { Los adolescentes } \\
\text { no conocen sus } \\
\text { derechos }\end{array}$ & 16 & 19.05 & 41 & 48.81 & 16 & 19.05 & 7 & 8.33 & 3 & 3.57 \\
\hline $\begin{array}{l}\text { Los adolescentes } \\
\text { conocen sus derechos } \\
\text { pero no sus obliga- } \\
\text { ciones }\end{array}$ & 40 & 47.62 & 31 & 36.90 & 7 & 8.33 & 4 & 4.76 & 1 & 1.19 \\
\hline $\begin{array}{l}\text { Los derechos de los } \\
\text { adolescentes han } \\
\text { disminuido la autori- } \\
\text { dad de los profesores }\end{array}$ & 39 & 46.43 & 25 & 29.76 & 11 & 13.10 & 8 & 9.52 & 1 & 1.19 \\
\hline $\begin{array}{l}\text { Los adolescentes } \\
\text { son rebeldes y deso- } \\
\text { bedientes }\end{array}$ & 30 & 35.71 & 40 & 47.62 & 8 & 9.52 & 5 & 5.95 & 1 & 1.19 \\
\hline $\begin{array}{l}\text { Los profesores no } \\
\text { conocen los derechos } \\
\text { de los adolescentes }\end{array}$ & 2 & 2.38 & 32 & 38.10 & 17 & 20.24 & 32 & 38.10 & 1 & 1.19 \\
\hline $\begin{array}{l}\text { Los adolescentes } \\
\text { quieren tomar } \\
\text { decisiones que no } \\
\text { les corresponden }\end{array}$ & 28 & 33.33 & 37 & 44.05 & 12 & 14.29 & 5 & 5.95 & 2 & 2.38 \\
\hline $\begin{array}{l}\text { La disciplina en el } \\
\text { salón de clases impone } \\
\text { medidas coercitivas } \\
\text { como necesario para } \\
\text { controlar el grupo }\end{array}$ & 18 & 21.43 & 31 & 36.90 & 16 & 19.05 & 15 & 17.86 & 2 & 2.38 \\
\hline
\end{tabular}

Fuente: elaboración propia. 
Tabla 5.Valores que muestran la posición de los docentes a que los estudiantes se organicen para exigir sus derechos

\begin{tabular}{|l|c|c|c|c|}
\hline $\begin{array}{c}\text { Etiqueta } \\
\text { de valor }\end{array}$ & Valor & Frecuencia & Porcentaje & $\begin{array}{c}\text { Porcentaje } \\
\text { Acumulado }\end{array}$ \\
\hline & .00 & 2 & 2.38 & 2.38 \\
\hline Mucho & 1.00 & 26 & 30.95 & 33.33 \\
\hline Algo & 2.00 & 36 & 42.86 & 76.19 \\
\hline Poco & 3.00 & 11 & 13.10 & 89.29 \\
\hline Nada & 4.00 & 7 & 8.33 & 97.62 \\
\hline No sé & 5.00 & 2 & 2.38 & 100.00 \\
\hline & Total & $\mathbf{8 4}$ & $\mathbf{1 0 0 . 0}$ & \\
\hline
\end{tabular}

Fuente: elaboración propia.

Tabla 6. La preparación de los docentes para promover los DD.HH., según ellos mismos

\begin{tabular}{|c|c|c|c|c|}
\hline $\begin{array}{c}\text { Etiqueta } \\
\text { de valor }\end{array}$ & Valor & Frecuencia & Porcentaje & $\begin{array}{c}\text { Porcentaje } \\
\text { Acumulado }\end{array}$ \\
\hline 1 & 0.0 & 0 & 0 & 0.0 \\
\hline 2 & 0.0 & 0 & 0 & 0.0 \\
\hline 3 & 2.00 & 1 & 1.19 & 2.38 \\
\hline 4 & 4.00 & 1 & 1.19 & 3.57 \\
\hline 5 & 5.00 & 4 & 4.76 & 8.33 \\
\hline 6 & 6.00 & 7 & 8.33 & 16.67 \\
\hline 7 & 7.00 & 19 & 22.62 & 39.29 \\
\hline 8 & 8.00 & 34 & 40.48 & 79.76 \\
\hline 9 & 9.00 & 5 & 5.95 & 85.71 \\
\hline 10 & 10.00 & 12 & 14.29 & 100.00 \\
\hline & Total & $\mathbf{8 4}$ & $\mathbf{1 0 0 . 0}$ & \\
\hline
\end{tabular}

Fuente: elaboración propia. 
rísticas de los adolescentes" (Profa. 26); "Porque en la materia de tutoría se promueven, así como transversalmente en todos los contenidos se dan a conocer los derechos, obligaciones y valores" (Profa. 08). Por su parte, el grupo de docentes que piensan que el conocimiento es menor a esas estimaciones arguye posiciones en este sentido: "Sólo los maestros que imparten materias como formación cívica y ética están más informados sobre eso. A los demás nos falta conocimiento" (Prof.14); "Desconocimiento al manual de convivencia" (Profa. 56); "En muchas ocasiones no tomamos en cuenta sus puntos de vista para trabajar e incluso algunas veces hay falta de respeto hacia ellos" (Prof. 12).

Como hemos venido observando, la promoción de los DD.HH. de los adolescentes en la escuela se relaciona con el grado de sensibilización de los profesores. Esto implica que los docentes deben recibir capacitación idónea para que se conviertan en agentes activos y responsables en su ejercicio efectivo. Empero, en una escala de cuatro categorías (siempre $=1$; frecuentemente $=2$; rara vez $=3$; nun$\mathrm{ca}=4$ ) podemos apreciar que esta tarea es deficitaria, pues la respuesta nunca se posiciona de manera significativa (Tabla 7).

\section{Discusión}

En este trabajo partimos del supuesto de que los profesores tienen un conocimiento marginal de la LGDDNA que moldea, en el fondo y en la forma, sus concepciones sobre los DD. HH. de los adolescentes. Los resultados nos permiten sostener que, con base en sus intuiciones, los docentes juzgan si los alumnos tienen o no capacidad para deliberar y proponer acciones que fomenten el ejercicio efectivo de los DD.HH. en la escuela. De cierta manera, esta posición cancela las oportunidades que puede haber en la interacción social de los jóvenes con los adultos

Tabla 7. Frecuencia mediante la cual los docentes reciben capacitación sobre los DD.HH. de sus alumnos

\begin{tabular}{|l|c|c|c|c|}
\hline $\begin{array}{c}\text { Etiqueta } \\
\text { de valor }\end{array}$ & Valor & Frecuencia & Porcentaje & $\begin{array}{c}\text { Porcentaje } \\
\text { Acumulado }\end{array}$ \\
\hline & .00 & 2 & 2.38 & 2.38 \\
\hline Siempre & 1.00 & 2 & 2.38 & 4.76 \\
\hline Frecuentemente & 2.00 & 9 & 10.71 & 15.48 \\
\hline Rara vez & 3.00 & 29 & 34.52 & 50.00 \\
\hline Nunca & 4.00 & 42 & 50.00 & 100.00 \\
\hline & Total & $\mathbf{8 4}$ & $\mathbf{1 0 0 . 0}$ & \\
\hline
\end{tabular}

Fuente: elaboración propia. 
y las instituciones (Silva, 2017). Asimismo, los datos nos permiten intuir que los entrevistados no tienen experiencia desde un enfoque de DD.HH. para resolver conflictos y situaciones que presentan los alumnos en el salón de clases. Esto a pesar de que ellos mismos reconocen que en sus escuelas se respetan "mucho" estos derechos, lo cual se constata cuando una gran mayoría asevera que nunca reciben cursos de capacitación en esta materia.

Dentro de los puntos de vista de los docentes encontramos el continuum de posiciones que se deslizan desde un punto $A$ hasta un punto B. Estos giros posicionales son contradictorios en términos prácticos, porque sostienen al mismo tiempo A y B sin percatarse de que las respuestas se contraponen. Este intento, quizá inconsciente, de reconciliar dos posturas contrarias evidencia que los docentes tienen poca claridad sobre los DD.HH. de los adolescentes.

Para ilustrar lo anterior, señalemos dos situaciones muy claras al respecto: (1) en primer lugar, argumentan que en la escuela se respetan mucho los DD.HH. de los adolescentes y ellos se consideran suficientemente preparados para promoverlos; en segundo lugar, no justifican algunas conductas que los estudiantes asumen en los salones de clase, las cuales están relacionadas, por ejemplo, con su derecho a construirse una identidad. (2) en primera instancia, arguyen que las opiniones de los alumnos deben tomarse en cuenta en las actividades escolares; en segunda instancia, suponen que los adolescentes son demasiado "adolescentes" para participar en dichas actividades, i.e., no los consideran suficientemente preparados para decir qué sí y qué no.

Así pues, si tomamos en cuenta lo establecido por la LGDDNA y confrontamos los giros posicionales de los docentes, en términos prácticos, podemos concluir que los profesores prestan poca atención al ejercicio efectivo de los DD.HH. de los jóvenes. Estas posiciones nos remiten al clásico problema de la institucionalización de los DD.HH. versus su impacto efectivo, tal como lo sostienen algunos estudiosos (Matus, 2013; Ansolabehere, Valdés \& Vázquez, 2015; Brito, 2016). En ese sentido, como afirma Magallón (2015), los derechos humanos son hechos, porque es en la puesta en práctica cuando se presentan una serie de acciones y omisiones que dificultan su disfrute. En el caso de los docentes, es razonable pensar, después de escucharlos, que tenemos suficientes elementos para afirmar que ellos, desde un punto de vista formal, aceptan y reconocen los DH de sus alumnos, pero dejan entrever el problema de su aplicación efectiva con las valoraciones que elaboran en relación a sus propias acciones. Aunque los resultados también permiten esgrimir, o por lo menos así lo inferimos, que los docentes desconocen el contenido de la LGDDNA.

Sin embargo, las anteriores contradicciones no son las únicas que encajan en estos giros posicionales. El tema de los DD.HH. también se queda en el ámbito de la retórica cuando un alto porcentaje de los docentes están de acuerdo en que los alumnos se organicen para exigir sus derechos, pero al mismo tiempo se oponen a que ellos tomen decisiones bajo el pretexto de "que no les corresponden". Este desplazamiento retórico se puede interpretar de dos maneras: (a) los profesores no aceptan realmente un involucramiento activo de sus alumnos en la construcción de los proyectos escolares, lo cual es su derecho y (b) no los consideran con 
la madurez apropiada para deliberar sobre sus derechos y obligaciones, así como de proponer soluciones responsables sobre asuntos de su competencia.

Esas son dos interpretaciones posibles, pero en el fondo ¿qué significados entrañan estos giros posicionales? Se puede señalar que reafirman el imaginario social sobre los estereotipos alrededor de los adolescentes (D'Aloisio, 2014), los que instituyen imágenes y comprensiones restringidas de apatía, desinterés y en algunos casos de peligrosidad (Tomasini \& Paulín, 2014).

Unido a lo anterior, encontramos consistentemente que los profesores no aprueban ciertos comportamientos dentro de la escuela, lo que les sirve de justificación suficiente para la expulsión de los alumnos infractores. Desde luego, en esos casos los docentes están obligados a explicar por qué no aceptan estas conductas de los jóvenes, tarea que requiere el despliegue de su imaginación para darse cuenta de que los adolescentes son en ciertos momentos más frágiles que en otros; por ejemplo, al momento de prohibir, castigar y señalar cuáles conductas son o no permisivas en el aula (Bázquez-Ruiz, 2013). Esta tarea, con certeza, los llevaría a evaluar cuáles comportamientos son aceptables desde un punto de vista moral y cuáles en el fondo son censurables, y para ello tienen que poner en juego su prudencia, pues un cometido de su labor es preocuparse por inducir actitudes que conduzcan a los adolescentes a ser buenas personas.

Rorty (1998) señala que el educador moral debe responder a la siguiente pregunta "¿por qué debo preocuparme por un extraño, una persona que no es de mi familia, una persona cuyas costumbres me parecen detestables?" (p.135). Siguiendo el argumento de Rorty podemos dar una respuesta: porque este joven en condiciones similares actuaría como lo haría mi hijo, o porque mi hijo podría estar en situaciones similares a las de este joven. Se nos podrá cuestionar que las cosas no son así de sencillas como se plantean en nuestras reflexiones; por supuesto que no lo son, ya que el ejercicio docente se da en dimensiones complejas (Porta, Aguirre \& Bazán, 2017). Sin embargo, está claro que los adolescentes son destinatarios de una serie de derechos que la escuela está obligada a conocer, reconocer y a poner en práctica de manera eficaz y sin interferencias. Por esta razón, se exige un compromiso virtuoso de los docentes, cuya disposición hemos de evaluar por sus intenciones, como a cualquier persona (Foot, 1994). En este sentido, no está demás que el mismo docente cuestione el sistema de enseñanza que proyecta en su práctica diaria (Díaz, 2017).

Con relación a la forma de contemplar a los adolescentes en tanto que agentes activos y responsables en el ejercicio de sus DD.HH., vemos una posición consistente en el juicio de los docentes, en la cual asocian que los jóvenes demandan derechos, pero no se asumen como sujetos de obligaciones. ¿Qué pasa cuando un discurso así se consolida? Pues pueden surgir, como en toda persona, la desconfianza y el temor a que su autoridad se vea amenazada (Nussbaum, 2010). Esto se relaciona con el imaginario extendido de que el posicionamiento de los $\mathrm{DD}$.HH. de los adolescentes altera la disciplina; por esta razón los docentes aprueban, mayoritariamente, que deben adoptarse medidas coercitivas que permitan el buen desarrollo de las actividades escolares. 
Estas medidas nos muestran, por una parte, la debilidad epistemológica que tienen los profesores sobre los DD.HH. de sus alumnos y, por otra, que sus posiciones están cargadas de un tinte emocional, porque en el fondo las preguntas cuestionan, en algún sentido, su quehacer educativo. Esto quedó de manifiesto cuando algunos de ellos expresaron ideas como las siguientes: "Es importante que también se hable de los derechos de los maestros, porque nosotros también los tenemos y de eso no se habla" (Profa. 34); "En todo esto, cuándo tomarán en cuenta nuestra opinión, también tenemos derechos" (Prof. 82).

Sin duda, las posiciones de los docentes nos permiten valorar que es necesaria la transformación de sus juicios acerca de la participación de los alumnos en la escuela, terminando de una vez por todas con el estereotipo del adolescente rebelde, difícil de tratar e incapaz de cumplir normas, que una gran mayoría de profesores sostiene. El argumento es un tanto simple: cuando voluntariamente 0 involuntariamente a los adolescentes no se les habilita o autoriza para hablar de cómo se pueden llevar a cabo los asuntos escolares, en el fondo se les niega su reconocimiento como sujetos de derechos, infligiendo a largo plazo un daño a la sociedad, porque estamos inhabilitando a los futuros promotores de los DD.HH. a que se conviertan en agentes activos en su defensa.

Una escuela educada en los DD.HH. no se da de manera espontánea, estamos convencidos de que se requiere poner atención especial en el diseño de estrategias cuidadosamente elaboradas que atraviesen las actividades cotidianas de la tarea escolar. Si pretendemos que los adolescentes sean agentes promotores de los DD.HH. debemos enseñarlos primero, a que los conozcan y, segundo, a que se asuman como defensores. Para esto es menester que los profesores se den cuenta que en muchas ocasiones sólo les corresponde escuchar las propuestas de los alumnos, lo que no implica en automático claudicar ante sus demandas. Puede haber más argumentos para, en la interlocución, sensibilizar a los jóvenes sobre sus derechos, mismos que deben ser relacionados con el planteamiento de sus obligaciones en el aula, porque incuestionablemente ser portador de derechos implica también ser portador de obligaciones.

Es por ello que no se trata de cultivar una cultura de los DD.HH., por más sublime que sea la tarea, sin que los adolescentes reflexionen que en el salón de clases todos debemos poner algo para tener una escuela educada; hablar de derechos conlleva una clara conexión con el deber y la responsabilidad (González, 2009). Esto implica que en todo proceso de deliberación sobre los derechos es conveniente saber escuchar para luego poder hablar. En términos de Lyotard (1998), en los procesos de interlocución y reconocimiento, el profesor tiene que aprender el lenguaje de los alumnos y éstos el lenguaje del profesor.

Finalmente, para poder deliberar sobre los DD.HH. en la escuela se requiere un conocimiento básico en esta materia por parte de los docentes, porque a ellos les corresponde provocar, sensibilizar y fomentar su discusión en el aula. Pero, si no conocen la LGDDNA, y si los DD.HH. nos les dicen nada, están condenados a repetir las mismas estrategias didácticas, y a conservar los mismos juicios de valor acerca de sus alumnos. En términos prácticos, se puede sostener que lo que no se conoce, no se puede ejercitar ni defender. 


\section{Conclusión}

El cuestionamiento que podemos hacer con relación a los DD.HH. de los adolescentes se focaliza en que las escuelas están, en diversos grados, al margen de su aplicación efectiva porque los profesores no reciben capacitación en esta materia, lo que implica, entre otras cosas, un desconocimiento de la LGDDNA y, por ende, de los protocolos a seguir cuando se presentan problemas que reclaman una atención oportuna y reflexiva con base en dicha ley.

Nos queda claro que no se trata de colocar los DD.HH. como dogmas de fe, como lo señala Guerrero (2015), ni tampoco convertirlos en criterios homogéneos y acabados que dificulten la aceptación de la diversidad. Esta última arista tiene serias implicaciones, pues demostraría nuestra incapacidad para atender los derechos de aquellos que, por diversas circunstancias, ajenas a ellos mismos, se encuentran todavía en condiciones de mayor vulnerabilidad. Pero sí es urgente una capacitación de los docentes que permita ir moldeando una infraestructura de los $\mathrm{DH}$ en el espacio escolar, porque de otra manera la escuela seguirá condenada a resolver los problemas de manera intuitiva y no con un conocimiento mínimo en la materia. Sin un ethos o cultura efectiva de los DD.HH. en la escuela estamos condenados a violentar las normas de la convivencia escolar.

La lección, por tanto, es seguir profundizando en este tema, porque los DD.HH. de los adolescentes en la escuela presentan, prima facie, un ejercicio deficitario. Sabemos que esto implica todo un proceso de sensibilización en el personal docente, proceso que debe contemplar, como condiciones necesarias, tres dimensiones: i) ver en cada estudiante a un posible interlocutor, ii) concebirlo como un semejante que tiene el derecho de hablar y iii) aprender a escucharlo y guardar silencio cuando habla. Estas dimensiones entrañan, entonces, el reconocimiento pleno del alumno como un Tú, es decir, como sujeto con facultades plenas para deliberar sobre temas de relevancia escolar; esta puede ser, indudablemente, una buena premisa para cultivar el respeto a los DD. $\mathrm{HH}$. de los adolescentes en la escuela.

Para concluir diremos que el presente trabajo es solamente un ejercicio exploratorio, y por tanto aproximativo a lo que sucede en un número limitado de escuelas públicas de la ciudad de Guadalajara; sin embargo, constituye un indicativo de cómo se está viviendo, en nuestro contexto, la promoción de los DD.HH. de los adolescentes a casi cuatro años de la promulgación de la LGDDNA. 


\section{Referencias bibliográficas}

Adolescentes, las que más desaparecen en la entidad (12 de noviembre de 2016). El Informador. Recuperado el 13 de noviembre de 2016, de http://www.informador.com.mx/ jalisco/2016/691609/6/adolescentes-las-que-mas-desaparecen-en-la-entidad.htm.

Amar, J., Madariaga, C. \& Macías, A. (2007). Infancia, familia y derechos humanos. Barranquilla, Colombia: Uninorte.

Ansolabehere, K. \& Navarrete, R. (2015). Derecho a la alimentación: difusión exitosa, impacto limitado. En K. Ansolabehere, F. Valdés \& D. Vázquez (Eds.), Los derechos humanos en América Latina (pp. 237-272). Bogotá: Universidad de los Andes/FLACSO.

Ansolabehere, K., Valdés, F. \& Vázquez D. (2015). Los derechos humanos en América Latina. Bogotá: Universidad de los Andes/FLACSO.

Bauman, Z. \& Donskis, L. (2015). Ceguera moral. La pérdida de sensibilidad en la modernidad líquida. México: Paidós.

Bázquez-Ruiz, F. (2013). Dialéctica de los derechos humanos. Madrid: Dykinson.

Brito, R. (2016). La noción de derechos humanos en la constitución mexicana. En L. R. Guerrero \& C. M. Pelayo (Coords.), 100 años de la constitución mexicana: de las garantías individuales a los derechos humanos (pp. 75-95). México: UNAM.

Cea, M.A. (2001). Metodología cuantitativa. Estrategias y técnicas de investigación social. Madrid: Síntesis.

D'Aloisio, F. (2014). Mirar el provenir a través de la secundaria: la concreción de proyectos biográficos, ¿una cuestión de voluntad individual? En H. Paulín \& M. Tomasini, Jóvenes y escuela: Relatos de una relación compleja (pp. 19-50). Córdoba: Brujas. Recuperado el 7 de febrero de 2018, de http://web.a.ebscohost.com.wdg.biblio.udg.mx:2048/ehost/ ebookviewer/ebook/bmxIYmtfXzgwMDE1MV9fQU41 ?sid=a3b4aeb1-974b-4703-acb7a77fc7256630@sessionmgr4010\&vid=7\&format=EB\&rid=1.

Díaz, C. M. (2017). Escuela, cultura y subjetividad: construcción de los significados, el imaginario y las representaciones del acto de educar. Diálogos pedagógicos, XV(30), 8292. DOI: http://dx.doi.org/10.22529/dp.2017.15(30)04.

Durkheim, E. (2002). La educación moral. Madrid: Morata.

Encuesta de Cohesión Social para la Prevención de la Violencia y la Delincuencia 2014 (2015). Recuperado el 2 de diciembre 2016, de http://www.beta.inegi.org.mx/contenidos/ proyectos/enchogares/especiales/ecopred/2014/doc/ecopred14_presentacion _ejecutiva.pdf.

Encuesta Nacional de la Juventud 2010 (2011). Recuperado el 7 de enero, de https:// www.imjuventud.gob.mx/imgs/uploads/Encuesta_Nacional_de_Juventud_2010__Resultados_Generales_18nov11.pdf.

Encuesta Nacional de Salud y Nutrición 2012 (2012). Recuperado el 6 diciembre de 2016, de https://ensanut.insp.mx/informes/ENSANUT2012ResultadosNacionales.pdf. 
Flick, U. (2007). Introducción a la investigación cualitativa. Madrid: Morata.

Fuentes, M.L., González, M. Padrón, M. \& Tapia, E. (2015). Conocimientos, ideas y representaciones acerca de niños, adolescentes y jóvenes. ¿Cambio o continuidad? Encuesta Nacional de Niños, Adolescentes y Jóvenes. Recuperado el 14 de agosto de 2017, de http:/ /www.losmexicanos.unam.mx/ninosadolescentesyjovenes/index.html.

Foot, P. (1994). Las virtudes y los vicios. México: UNAM.

González, R. (2009). Educar en los deberes y las responsabilidades en la sociedad de los derechos y las libertades. En J. A. Ibáñez-Martín (Coord.), Educación, conocimiento y justicia (pp. 281-294). Madrid: Dykinson.

González, M. (2016). La constitución mexicana vista a la luz de los modelos de tratamiento jurídico de la infancia y adolescencia. En R. Guerrero \& C. Pelayo, 100 años de la constitución mexicana: de las garantías individuales a los derechos humanos (pp. 339-356). MéxiCO: UNAM.

Guerrero, A. L. (2015). Dignidad intercultural y su fundamentación. En J. Olvera, J. Olvera \& A. L. Guerrero (Coords.), Derechos Humanos y genealogía de la dignidad en América Latina (pp. 305-325). México: UNAM/M.A. Porrúa.

Hernández, R., Fernández, C. \& Baptista, P. (2014). Metodología de la investigación. México: McGraw-Hill.

Instituto Nacional de Salud Pública y UNICEF México (2016). Encuesta Nacional de Niños, Niñas y Mujeres 2015 - Encuesta de Indicadores Múltiples por Conglomerados 2015, Informe Final. México: Instituto Nacional de Salud Pública y UNICEF México. Recuperado de https://www.insp.mx/enim2015/informe-final-enim.html.

Lyotard, J. (1998). Los derechos de los otros. En A. Shute \& S. Hurley (Eds.), De los derechos humanos (pp.137-145). Madrid: Trotta.

Magallón, M (2015). Filosofía hermenéutica e interculturalidad de los derechos humanos en la modernidad alternativa radical. En J. Olvera, J. Olvera, A. L. Guerrero (Coords.), Derechos Humanos y genealogía de la dignidad en América Latina (pp. 45-75). México: UNAM/ M.A. Porrúa.

Matus, E. (2013). México ante la restitución internacional de menores. México: UNAM.

México, Comisión Nacional de los Derechos Humanos (2015). Ley General de los Derechos de Niñas, Niños y Adolescentes. Recuperado el 23 de julio de 2017, de http:// 200.33.14.34:1033/archivos/pdfs/lib_LeyGralCuidadoInfantil.pdf.

Nussbaum, M. (2010). Libertad de conciencia. Contra los fanatismos. México: Tusquets.

Organización de las Naciones Unidas, México (2008). Propuesta de reforma constitucional en materia de derechos humanos, elaborada por las organizaciones de la sociedad civil y por académicas y académicos especialistas en derechos humanos. México: Naciones Unidas México. Recuperado 31 de julio de 2017 de https://www.hchr.org.mx/images/doc_pub/ propuestareformaconst.pdf.

Páramo, P. (2013). La investigación en ciencias sociales: estrategias de investigación. Bogotá: Universidad Piloto de Colombia. 
Porta, L. Aguirre, J. \& Bazán, S. (2017). La práctica docente en los profesores memorables. Reflexividad, narrativa y sentidos vitales. Diálogos pedagógicos, XV(30), 15-36. DOI: http://dx.doi.org/10.22529/dp.2017.15(30)01

Ragin, CH. (2007). La construcción de la investigación social. Bogotá: Siglo del hombre.

Romney A.K., Weller S.C. \& Batchelder W.H. (1986). Culture as consensus. A theory of culture and informant accuracy. American Anthropologist, 81(2), 313-338. Recuperado el 2 de septiembre de 2017, de https://www.bebr.ufl.edu/sites/default/files/ Culture\%20as $\% 20$ Consensus.pdf.

Rorty, R. (1998). Derechos humanos: racionalidad y sentimentalidad. En A. Shute \& S. Hurley (Eds.), De los derechos humanos (pp. 117-136). Madrid: Trotta.

Silva, D. (2017). Experiencia narrativa. Adolescentes institucionalizados por protección [Libro electrónico]. Barcelona: UOC.

Tomasini, M. \& Paulín, H. (2014). Introducción. En H. Paulín \& M. Tomasini (Coords.). Jóvenes y escuela: Relatos de una relación compleja (pp. 5-17). Córdoba: Brujas. Recuperado el 28 de febrero de 2018, de http://web.a.ebscohost.com.wdg.biblio.udg.mx:2048/ehost/ ebookviewer/ebook/bmxlYmtfXzgwMDE1MV9fQU41 ?sid=a3b4aeb1-974b-4703-acb7a77fc7256630@sessionmgr4010\&vid=7\&format=EB\&rid=1.

Touraine, A. (1997). ¿Podremos vivir juntos? [libro electrónico]. Buenos Aires: Fondo de Cultura Económica.

Weber, M. (2009). La "objetividad" del conocimiento en la ciencia social y en la política social. Madrid: Alianza.

Yuni, J. A. \& Urbano, C. A. (2014). Técnicas para investigar: recursos metodológicos para la preparación de proyectos de investigación. Córdoba: Brujas. 